\title{
Analysis of local covariance functions applied to GOCE satellite gravity gradiometry data
}

\author{
Branislav Hábel $^{1}$ (D) Juraj Janák ${ }^{1}$
}

Received: 20 February 2017/Accepted: 12 October 2017/Published online: 31 October 2017

(C) Akadémiai Kiadó 2017

\begin{abstract}
GOCE Level 2 products of corrected gravity gradients in Local North-Oriented Frame were used in this study. We analyzed four accurately measured elements of the gravity tensor, which were transformed to disturbing gravitational gradients. The investigation was carried out in the restricted region of dimension $20^{\circ} \times 20^{\circ}$ covering the south part of Europe. We applied several types of analytical covariance functions in a local approximation, which have the best fit to the empirical covariances calculated from the disturbing gravitational gradients in particular sub-regions. At first, we have investigated four different types of the 1-dimensional covariance function. Obtained results show that the Gaussian covariance function approximates the empirical covariances the best from tested functions. Moreover, a time stability of calculated parameters of the covariance functions was studied by assuming GOCE data from different time periods. In the second experiment, we have compared two types of the 2-dimensional covariance function, which also enables a spatial stochastic modeling. The second study revealed that the least-squares collocation using the 2-dimensional local covariance function can produce the local grid of GOCE disturbing gravitational gradients directly from GOCE Level 2 products right below GOCE orbit, which in general fits well with the recent Earth's global gravity field models and might have some advantages. Such local grids can be useful for specific tasks, e.g. mutual comparing of GOCE data collected during particular time periods.
\end{abstract}

Keywords Gravity field · Local covariance function · GOCE · Least-squares collocation

Branislav Hábel

branislav.habel@stuba.sk

Juraj Janák

juraj.janak@stuba.sk

1 Department of Theoretical Geodesy, Faculty of Civil Engineering, Slovak University of Technology in Bratislava, Radlinského 11, 81005 Bratislava, Slovakia 


\section{Introduction}

The quantities of the Earth's gravity field are specified by the spatial correlation, which is widely used in applications of physical geodesy and geophysics. In general, a correlation indicates some form of similarity relation between two observables with tendency to have about same size and sign. If we consider the correlation as a function of position or time, we obtain a covariance function, which provides the basis for stochastic methods of regional and global gravity field modelling (Heiskanen and Moritz 1967). The most used least-squares prediction method in physical geodesy is called least-squares collocation, where the covariance function is a crucial ingredient. It offers a powerful tool for gravity field prediction, filtering, and parameter estimation (Tscherning 2010) and is also able to combine different kinds of terrestrial and satellite data. Produced local (regional) and global grids may be then used for other research, e.g. estimation of spherical harmonic coefficients derived by numerical integration.

The covariance functions can take a local and a global form (Moritz 1980). For the global covariance function, the data have to be distributed over the whole Earth to describe the stochastic properties of observables throughout the whole spectral range. Evaluation of the global covariance function requires more computational effort and large sets of data. Various authors have been dealing with the construction and application of global covariance function, e.g. Tscherning (1976), Rapp (1978), Pail et al. (2011) and Gatti et al. (2013). For some purposes, the local covariance function is more suitable rather than the global one. We are restricted only over a limited area instead of over the whole Earth. This approach has several advantages like handling with reduced amount of interpreted data, simpler practical determination and ensuring less computational effort. It allows carrying out more detailed studies, e.g. the interpolation problem and filtering. Local grids produced by suggested method may be more flexible than several existing global grids, e.g. Bouman et al. (2016), in terms of time span of used data or altitude. However, a true shape of the covariance function is rarely known and it depends on the region of calculation. Therefore, it must be derived directly from measured data (Jekeli 2010). The local grid is also characterized by the limited spectral resolution than the global one.

The aim of our contribution is to investigate the statistical behaviour of particular components of the disturbing gravitational tensor derived from GOCE (Gravity field and steady-state Ocean Circulation Explorer) on-orbit data. It was the first satellite gravity gradiometry (SGG) mission in the history, which was developed and led by the European Space Agency (ESA). GOCE satellite was launched on 17 March 2009 from the Plesetsk Cosmodrome in Russia. The mission finished after 4 years and 8 months on 11 November 2013, when a design life of the satellite was exceeded of about 20 months. A primary objective of GOCE was to improve a global model of the Earth's gravity field and a geoid, both in spatial detail and accuracy, especially over the ocean (ESA 1999). Nowadays, GOCE still brings a number of applications in oceanography, geodesy, solid earth physics and many other disciplines. An extended life time and an extremely low orbit of the satellite resulted in very interesting and unique data. The spacecraft was equipped by the electrostatic gravity gradiometer with a set of three pairs of orthogonally mounted 3-axis accelerometers. It allowed to measure gravity gradients in all directions. However, only four components $\left(V_{X X}, V_{Y Y}, V_{Z Z}\right.$, and $\left.V_{X Z}\right)$ of the tensor were measured with ultra-high precision due to the arrangement of accelerometers. A position of the satellite was controlled using the on-board Global Positioning System receiver by the Satellite-to-Satellite Tracking principle. The orientation of the satellite with regard to inertial reference frame was monitored by the star-trackers. Three levels of data products are available. Level 0 
includes raw data gathered by GOCE satellite. Level 1b products consist of calibrated gravity gradients measured in the gradiometer reference frame including the orbit data. These were further processed by the European GOCE Gravity Consortium (E-GGC) to Level 2 products of corrected gravity gradients in different reference frames. More details about GOCE mission and provided products can be found in Gruber et al. (2010).

In this study, we have focused only on local covariance functions in the planar approximation. By the term 'covariance model' we will understand the analytical covariance function together with a small numbers of corresponding parameters. We will test different types of planar covariance models used in physical geodesy and examine both the spatial and the time dependencies of their essential parameters. Proving the usefulness of the local covariance function, we will apply the least-squares collocation method for the interpolation of the largest component of disturbing gravitational tensor to a regular grid and compare it with the recent Earth's global gravity field models: TIM R5 (Brockmann et al. 2014), EIGEN-6C4 (Förste et al. 2014), and EGM2008 (Pavlis et al. 2012).

\section{Local covariance functions}

We have restricted our study to local covariance functions described in Moritz (1980), which are well-known in physical geodesy. Let us suppose, for simplicity, that GOCE satellite is moving in the circular orbit above the reference sphere $R$, which lies on the spherical surface with the radius $R_{1}$ (see Fig. 1). Then, a local structure of the covariance function assumes a planar approximation, where the spherical surface is replaced by its tangent plane around the position of the satellite $P$. If there are all positions of measured data points situated on the tangent plane, we can estimate the 1-dimensional covariance model. In this case, a stochastic dependence in the vertical direction, if exists, is neglected. Such homogenous and isotropic covariance function depends only on planar distances $\rho$ between data points that are calculated from the spherical distances $\psi$ using a simple formula:

$$
\rho=R_{1} \tan \psi \approx R_{1} \psi
$$

Behavior of the local covariance function can be characterized by three essential parameters $\left(C_{0}, \xi, \chi\right)$. A variance $C_{0}$ corresponds to the covariance for the zero planar distance $\rho$. A correlation length $\xi$ represents the planar distance $\rho$ corresponding to one half of the variance $C_{0}$. The last dimensionless parameter $\chi$ is related to the curvature of the

Fig. 1 Planar approximation of the spherical surface with the radius $R_{1}$

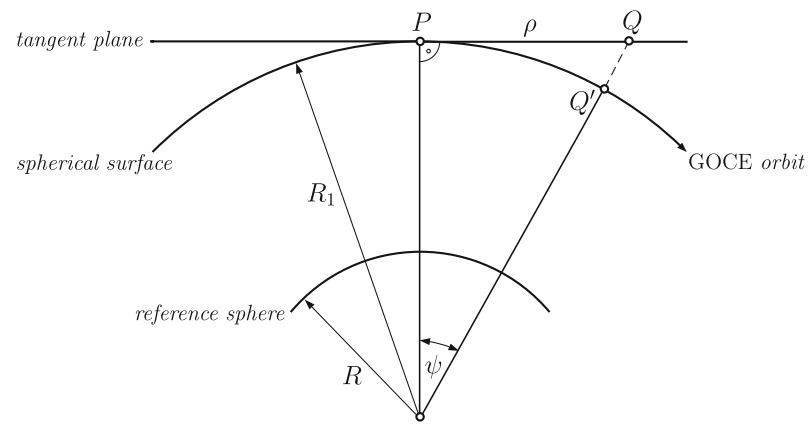


covariance function at the distance $\rho=0$ (Moritz 1980). We have tested four different types of the 1-dimensional positive definite functions shown in Table 1.

According to Moritz (1980), some 1-dimensional covariance functions can be extended to outer space by simple modification, where the altitude of data points is $z>0$. Such modeling is able to express the stochastic dependence in both the horizontal and the vertical direction between data points $P(z)$ and $Q\left(z^{\prime}\right)$ located above the approximation plane. Altitude $z$ of particular point can be calculated as a difference between its radial distance $r$ and the radius of approximation sphere $R_{1}$. Two types of the 2-dimensional covariance function labeled as CF5 and CF6 are listed in Table 2. Constants $A, B, b$ and $m$ mentioned in Tables 1 and 2 represent additional parameters, which enable to derive the essential parameters $\xi$ and $\chi$.

The essential parameters of covariance functions are either applied as theoretical values or derived from the given data. The second approach is more frequent and requires a construction of the empirical covariance function (ECF) as the first. ECF is a point-wise function and its shape primarily depends on the input data, but also on the chosen number of bins dividing data into a set of equally sized intervals. In 1-dimensional case and irregularly spaced data, the empirical covariances can be computed as:

$$
\operatorname{cov}\left(\rho_{a} \leq \rho_{i, j}<\rho_{b}\right)=\frac{1}{n} \sum_{k=1}^{n}\left\{\left(l_{i}-\bar{l}\right)\left(l_{j}-\bar{l}\right)\right\}_{k},
$$

where $l_{i}$ and $l_{j}$ are measurements (e.g. gravity gradients), which correspond to the planar distances $\rho_{i, j}$ between points $i, j$ and satisfy conditions $\rho_{i, j} \in\left\langle\rho_{a}, \rho_{b}\right)$, where $\rho_{a}$ and $\rho_{b}$ are the limits of the particular bin. The number of all pairs $k$ of points in data set satisfying that condition is denoted as $n$. Taken into account stationarity of the covariance function, measured quantities are centered, i.e. reduced by the mean value $\bar{l}$. This process of centration can be supplemented by a subtraction of normal gravity field in the sense of physical geodesy. Number of calculated empirical covariances, which corresponds to the number of bins, usually exceeds the number of unknown parameters of their analytical expression and allows applying the least-squares approach. In 2-dimensional case, the empirical covariance also depends on the sum of altitudes $z+z^{\prime}$ :

$$
\operatorname{cov}\left(\rho_{a} \leq \rho_{i, j}<\rho_{b},\left(z+z^{\prime}\right)_{a} \leq\left(z+z^{\prime}\right)_{i, j}<\left(z+z^{\prime}\right)_{b}\right)=\frac{1}{n} \sum_{k=1}^{n}\left\{\left(l_{i}-\bar{l}\right)\left(l_{j}-\bar{l}\right)\right\}_{k},
$$

where the measurements $l_{i}$ and $l_{j}$ also satisfy the second condition $\left(z+z^{\prime}\right)_{i, j} \in$ $\left\langle\left(z+z^{\prime}\right)_{a},\left(z+z^{\prime}\right)_{b}\right)$ with the limits $\left(z+z^{\prime}\right)_{a}$ and $\left(z+z^{\prime}\right)_{b}$ of particular bin.

Table 1 The 1-dimensional local covariance functions (Moritz 1980)

\begin{tabular}{llll}
\hline Covariance function & Expression length & Correlation & Curvature parameter \\
\hline CF1: Gaussian & $\operatorname{cov}(\rho)=C_{0} \exp \left(-A^{2} \rho^{2}\right)$ & $\xi=\frac{1}{A} \sqrt{\ln 2}$ & $2 \ln 2$ \\
CF2: with $m=2$ (Hirvonen's) & $\operatorname{cov}(\rho)=\frac{C_{0}}{\left(1+B^{2} \rho^{2}\right)^{2}}$ & $\xi=\frac{\sqrt{2^{1 / 2}-1}}{B}$ & $4\left(2^{1 / 2}-1\right)$ \\
CF3: with $m=1 / 2$ & $\operatorname{cov}(\rho)=\frac{C_{0}}{\left(1+B^{2} \rho^{2}\right)^{1 / 2}}$ & $\xi=\frac{\sqrt{3}}{B}$ & 3 \\
CF4: with $m=3 / 2$ & $\operatorname{cov}(\rho)=\frac{C_{0}}{\left(1+B^{2} \rho^{2}\right)^{3 / 2}}$ & $\xi=\frac{\sqrt{2^{2 / 3}-1}}{B}$ & $3\left(2^{2 / 3}-1\right)$ \\
\hline
\end{tabular}


Table 2 The 2-dimensional local covariance functions (Moritz 1980)

\begin{tabular}{llll}
\hline Covariance function & Expression length & Correlation & Curvature parameter \\
\hline CF5: with $m=1 / 2$ & $\operatorname{cov}(P, Q)=\frac{C_{0} b}{\left(\rho^{2}+\left(z+z^{\prime}+b\right)^{2}\right)^{1 / 2}}$ & $\xi=b \sqrt{3}$ & 3 \\
CF6: with $m=3 / 2$ & $\operatorname{cov}(P, Q)=\frac{C_{0} b^{2}\left(z+z^{\prime}+b\right)}{\left(\rho^{2}+\left(z+z^{\prime}+b\right)^{2}\right)^{3 / 2}}$ & $\xi=b \sqrt{2^{2 / 3}-1}$ & $3\left(2^{2 / 3}-1\right)$ \\
\hline
\end{tabular}

\section{Data preparation}

As mentioned above, we have analyzed the corrected gravity gradients of GOCE Level 2 products (EGG_TRF_2) available via ESA GOCE Virtual Archive website (http://eovirtual-archive1.esa.int/Index.html). Calibrated gradients in these products are given in Local-North Oriented Frame (LNOF). Geocentric position of the GOCE center of mass is defined by its spherical latitude $\phi$, longitude $\lambda$ and a radial distance $r$ for a particular observation epoch. This enables to calculate the spherical distances $\psi$ between the pairs of data points and the altitudes above the approximation plane eventually. For more information about the EGG_TRF_2 products and the definition of LNOF system, see Gruber et al. (2010).

Our experiment has focused on four accurately measured components of the gravity tensor $\left(V_{X X}, V_{Y Y}, V_{Z Z}\right.$, and $\left.V_{X Z}\right)$. All gradients flagged as outliers were excluded from further analysis. A region of interest $\left(20^{\circ} \times 20^{\circ}\right)$ covers the south part of Europe and is restricted to $\phi \in\left\langle 30^{\circ} \mathrm{N}, 50^{\circ} \mathrm{N}\right\rangle$, and $\lambda \in\left\langle 10^{\circ} \mathrm{E}, 30^{\circ} \mathrm{E}\right\rangle$. To study the behavior of local covariance functions in different areas, we have divided this region into four sub-regions of $10^{\circ} \times 10^{\circ}$ labeled as R1, R2, R3 and R4 (see Fig. 2). Measured quantities should be corrected for a systematic part of gravity field before evaluation of empirical covariances. Therefore, the gravity gradients were transformed to disturbing gravitational gradients $T_{X X}$, $T_{Y Y}, T_{Z Z}$, and $T_{X Z}$. These are obtained from the observed gravity tensor by subtraction of corresponding elements of normal gravity tensor by 'Normal tensor' software (Šprlák 2012) using GRS-80 reference ellipsoid. Calculated gradients in the test region are depicted in Fig. 2. Measurements from 19 January 2011 to 31 May 2011 were used for the visualization. Adjacent sub-regions are characterized by a different pattern of disturbing gravitational gradients. The amplitudes vary from $-1.5 \mathrm{E}$ to $1.0 \mathrm{E}(\mathrm{E}=$ Eötvös, $1 \mathrm{E}=$ $10^{-9} \mathrm{~s}^{-2}$ ) and are the largest for $T_{\mathrm{ZZ}}$ component.

\section{Experiment with the 1-dimensional local covariance functions}

The first experiment investigates performance of the 1-dimensional covariance functions $\mathrm{CF} 1, \mathrm{CF} 2, \mathrm{CF} 3$ and CF4 presented in Table 1. Let us assume a simplified situation, where GOCE satellite is moving on the spherical surface above the Earth. We consider that the radius of such sphere is equal to $R_{1}=6628 \mathrm{~km}$, what corresponds to the mean radial distance of the satellite in the region of interest. Then, all data points should have zero altitudes $(z=0)$ above the tangent plane, which locally approximates the sphere.

For all sub-regions and four components of the disturbing gravitational tensor the empirical covariances were evaluated according to Eq. (2). The planar distances $\rho$ were divided into 25 equally spaced bins in the range from 0 to $1000 \mathrm{~km}$. We have considered 

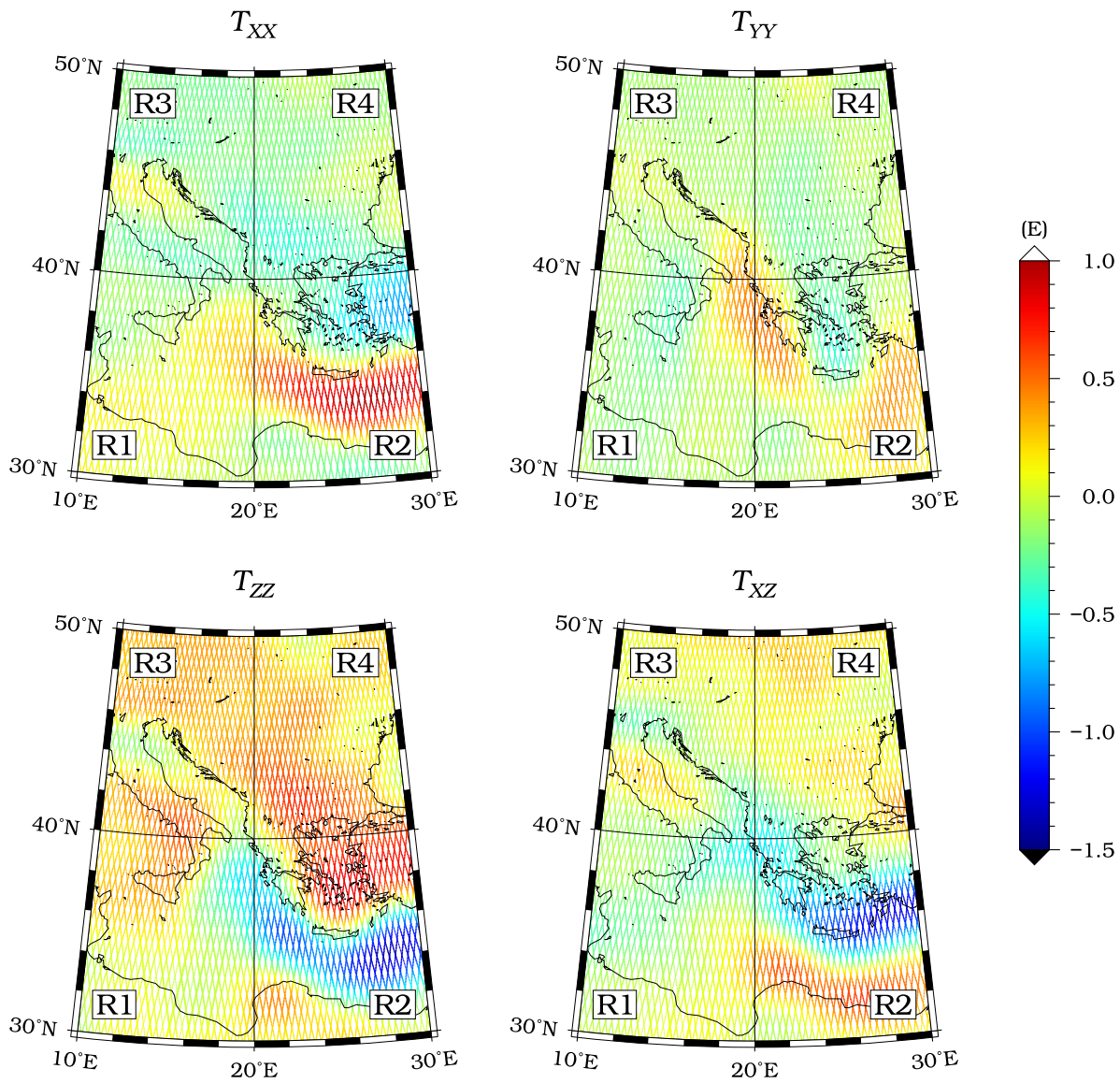

Fig. 2 The region of interest divided into four sub-regions and scatter plot of analyzed components of the disturbing gravitational tensor along the GOCE orbit

measurements from 19 January 2011 to 28 February 2011. ECF of $T_{Z Z}$ component is depicted in Fig. 3. Only positive empirical covariances are displayed as the local covariance model is expressed by the positive definite function according to covariance function theory (Moritz 1980). In addition, we should not overstep the correlation length within the interpolation procedure, where all empirical covariances get positive values.

Variances and correlation lengths for the 1-dimensional models from Table 1 were estimated using the least-squares approach. In order to asses how well the estimated model approximates the empirical covariances, the coefficient of determination $R s q$ is calculated as (Rousseeuw and Leroy 1987):

$$
R s q=1-\frac{\sum_{i=1}^{N}\left(y_{i}-\hat{y}_{i}\right)^{2}}{\sum_{i=1}^{N}\left(y_{i}-\bar{y}\right)^{2}},
$$

where the value $y_{i}$ represents positive empirical covariance, $\hat{y}_{i}$ is its least-squares estimate, and $\bar{y}$ is the mean value of $y$. Derived analytical covariance models for $T_{Z Z}$ component are also shown in Fig. 3. The best estimates are highlighted in bold. It is clearly visible that Gaussian covariance model $\mathrm{CF} 1$ has the best performance in all sub-regions. However, 

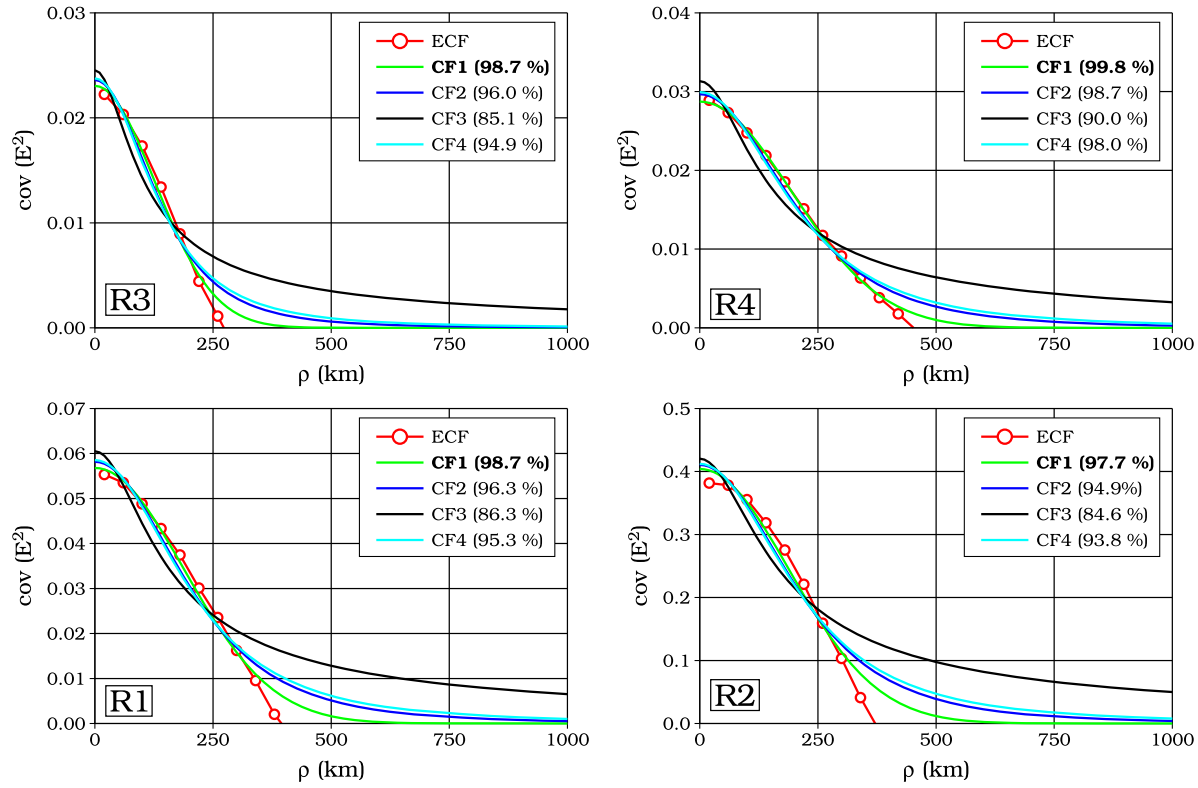

Fig. 3 The empirical covariance function of $T_{Z Z}$ component calculated in four sub-regions and approximated by analytical covariance models CF1, CF2, CF3 and CF4 with the corresponding coefficient of determination $R s q$ (in brackets)

differences between the particular models are almost negligible up to the correlation length, except for CF3 model. For completeness, mean essential parameters from four estimated models were calculated. Results involving all analyzed components of the disturbing gravitational tensor are summarized in Table 3 . It is not surprising that the estimates differ from one region to another, mainly for the mean variance $\overline{C_{0}}$. The highest values are typical for the sub-region $\mathrm{R} 2$ due to the most distinctive variation of disturbing

Table 3 Mean variances, correlation lengths and coefficients of determination calculated from the estimated covariance models $\mathrm{CF} 1, \mathrm{CF} 2, \mathrm{CF} 3$ and CF4 (the 1-dimensional case)

\begin{tabular}{llllll}
\hline Sub-reg. & Param. & $T_{X X}$ & $T_{Y Y}$ & $T_{Z Z}$ & $T_{X Z}$ \\
\hline R1 & $\overline{C_{0}}\left(\mathrm{E}^{2}\right)$ & 0.016 & 0.027 & 0.059 & 0.026 \\
& $\bar{\xi}(\mathrm{km})$ & 204.4 & 186.4 & 205.8 & 229.5 \\
& $R \bar{s} q(\%)$ & 95.9 & 95.5 & 94.2 & 96.4 \\
& $\overline{C_{0}}\left(\mathrm{E}^{2}\right)$ & 0.235 & 0.053 & 0.412 & 0.284 \\
$\mathrm{R} 2$ & $\bar{\xi}(\mathrm{km})$ & 215.7 & 186.8 & 213.9 & 222.0 \\
& $R \bar{s} q(\%)$ & 92.6 & 93.5 & 92.7 & 91.5 \\
& $\overline{C_{0}}\left(\mathrm{E}^{2}\right)$ & 0.012 & 0.008 & 0.024 & 0.021 \\
$\mathrm{R} 3$ & $\bar{\xi}(\mathrm{km})$ & 145.9 & 128.4 & 137.8 & 138.1 \\
& $R \bar{s} q(\%)$ & 93.3 & 96.2 & 93.7 & 95.1 \\
& $\overline{C_{0}}\left(\mathrm{E}^{2}\right)$ & 0.014 & 0.006 & 0.030 & 0.021 \\
$\mathrm{R} 4$ & $\bar{\xi}(\mathrm{km})$ & 199.6 & 171.5 & 206.6 & 166.1 \\
& $R \bar{s} q(\%)$ & 96.4 & 96.8 & 96.6 & 96.3 \\
\hline
\end{tabular}


gravitational gradients. Lower variances occur in the sub-regions R3 and R4. Correlation lengths vary between $128 \mathrm{~km}$ and $230 \mathrm{~km}$ depending on the sub-region.

Furthermore, we were interested in time stability of calculated covariance functions. Our aim was to study the sensitivity of estimated parameters to the changes of GOCE satellite orbits and to other phenomena, which affect measured gradients (e.g. mass redistribution). We have followed the same strategy as described above. The Gaussian covariance model CF1 showing the best performance in the 1-dimensional case has been applied to this study. We have examined $T_{Z Z}$ component of the disturbing gravitational tensor in all sub-regions. The radius of the approximation sphere remained unchanged $(6628 \mathrm{~km})$. ECF has been calculated from data collected in 6 two-month intervals during the year 2011. Parameters obtained by the least-squares method are listed in Table 4. Value denoted as $h$ is the mean altitude of GOCE satellite above GRS-80 reference ellipsoid in the particular sub-region. We assume that the variance will be increasing by decreasing altitude of GOCE orbit, and vice versa. This fact is clearly visible in Table 4. Anomalous behavior occurs between time intervals of 07-08/2011, 09-10/2011 and 11-12/2011, especially in the sub-regions R1 and R2. However, the estimated parameters do not change dramatically within the particular sub-region. Disparity of variances $C_{0}$ reaches a few $\mathrm{mE}^{2}$. This could be assigned mainly to satellite orbit changes. Therefore, the correct modeling of covariances requires consideration of different satellite altitudes above the approximation sphere and the tangent plane, respectively. However, the interpretation of this problem is not straightforward and further analyses would be required to formulate some clear conclusion.

Table 4 The estimated parameters of the covariance function CF1 (Gaussian) for $T_{Z Z}$ component

\begin{tabular}{lllllllll}
\hline Sub-reg. & Param. & $01-02$ & $03-04$ & $05-06$ & $07-08$ & $09-10$ & $11-12$ & Mean \\
\hline R1 & $C_{0}\left(\mathrm{E}^{2}\right)$ & 0.057 & 0.050 & 0.052 & 0.048 & 0.044 & 0.049 & 0.050 \\
& $\xi(\mathrm{km})$ & 220.8 & 207.9 & 203.1 & 208.3 & 207.2 & 208.6 & 209.3 \\
& $h(\mathrm{~km})$ & 261.4 & 262.2 & 262.4 & 261.5 & 261.7 & 262.5 & 262.0 \\
& $C_{0}\left(\mathrm{E}^{2}\right)$ & 0.404 & 0.384 & 0.383 & 0.378 & 0.405 & 0.377 & 0.388 \\
$\mathrm{R} 2$ & $\xi(\mathrm{km})$ & 221.5 & 216.4 & 218.5 & 214.5 & 221.2 & 215.9 & 218.0 \\
& $h(\mathrm{~km})$ & 261.2 & 262.2 & 262.7 & 261.3 & 261.9 & 262.7 & 262.0 \\
& $C_{0}\left(\mathrm{E}^{2}\right)$ & 0.023 & 0.021 & 0.021 & 0.022 & 0.023 & 0.022 & 0.022 \\
$\mathrm{R} 3$ & $\xi(\mathrm{km})$ & 148.6 & 146.9 & 145.6 & 146.7 & 148.6 & 148.2 & 147.4 \\
& $h(\mathrm{~km})$ & 263.3 & 264.1 & 264.2 & 263.3 & 263.5 & 264.4 & 263.8 \\
& $C_{0}\left(\mathrm{E}^{2}\right)$ & 0.029 & 0.028 & 0.027 & 0.028 & 0.027 & 0.026 & 0.027 \\
$\mathrm{R} 4$ & $\xi(\mathrm{km})$ & 227.2 & 228.5 & 225.3 & 229.1 & 228.6 & 226.2 & 227.5 \\
& $h(\mathrm{~km})$ & 262.7 & 264.1 & 264.8 & 262.9 & 263.8 & 264.9 & 263.9 \\
\hline
\end{tabular}

Numbers in the header row represent particular months of the year 2011 


\section{Experiment with the 2-dimensional local covariance functions}

As stated previously, the evaluated 1-dimensional covariance function can take a different shape for various measurement cycles, even in the same region. The main reason is that the covariance function does not take into account the altitudes of data points above the spherical surface $R_{1}$. Therefore, such model is not very suitable for SGG data. This disadvantage supersedes the 2-dimensional covariance function, which also enables spatial stochastic modeling.

An analysis has been made in the same region of interest as depicted in Fig. 2. Our goal was to examine stochastic dependencies of disturbing gravitational gradients in both the horizontal and the vertical direction. For this purpose, we have applied SGG data from the last measurement cycles collected from 1 June 2012 to 19 October 2013, when GOCE satellite has been stepwise decreasing its altitude. The radius of the approximation sphere of $R_{1}=6595 \mathrm{~km}$ was chosen in such a way that all data points are situated above this sphere $(z>0)$. In this case, both the planar distances $\rho$ and the corresponding sum of altitudes $z+z^{\prime}$ were distributed into ten equally spaced bins. Figure 4 a shows ECF of $T_{Z Z}$ component computed from Eq. (3) and corresponding to the sub-region R1 in two different views. It is possible to see a clear trend of covariance decreasing with increasing of both the planar distances $\rho$ and the sum of altitudes $z+z^{\prime}$, as shown in Figure 4b, c. Once again, only positive empirical covariances are displayed.
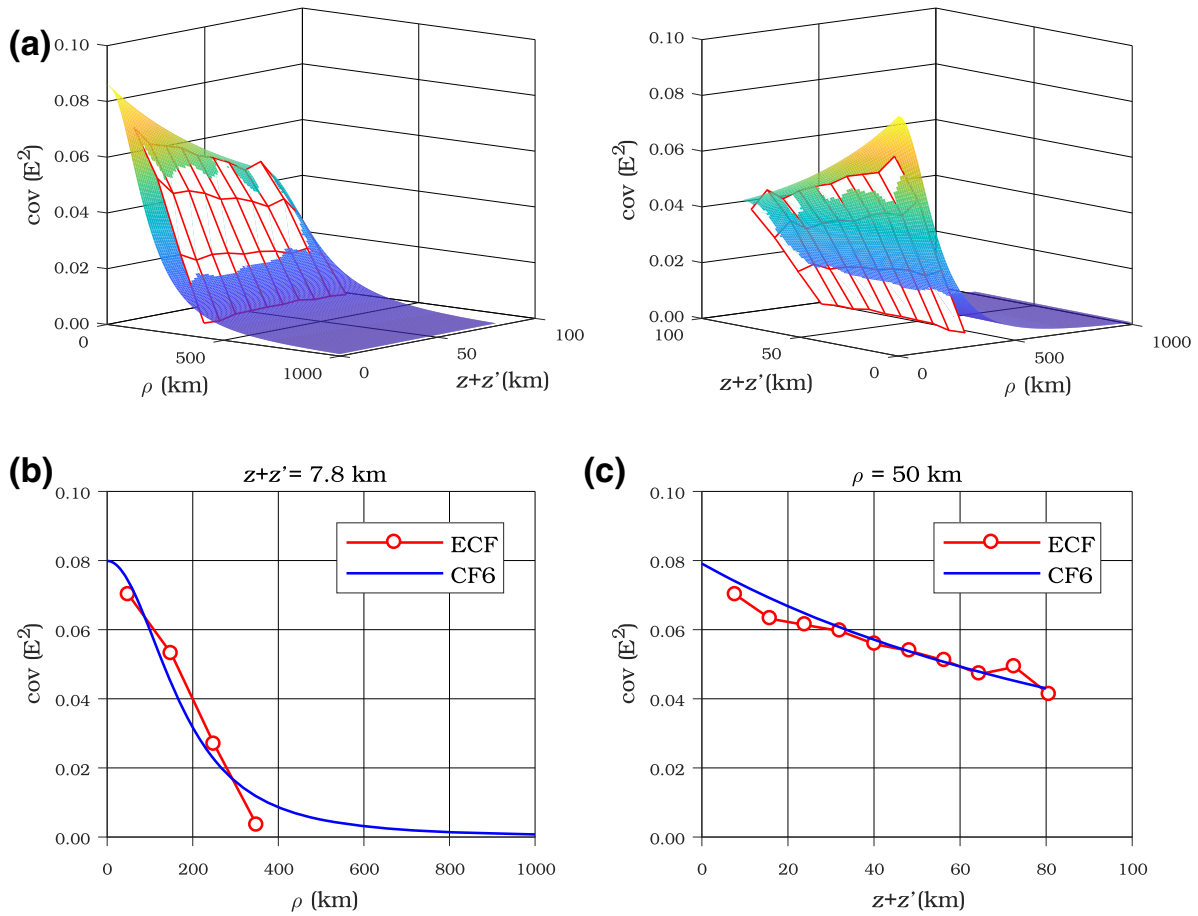

Fig. 4 a Two different views of the empirical covariance function of $T_{Z Z}$ component calculated in the subregion R1 (red mesh) approximated by the 2-dimensional covariance model CF6 (coloured surface), b vertical profile at $z+z^{\prime}=7.8 \mathrm{~km}$ and $\mathbf{c}$ vertical profile at $\rho=50 \mathrm{~km}$ 
Empirical covariances were fitted by two analytical covariance models CF5 and CF6 described in Table 2. Essential parameters, as variances $C_{0}$ and correlation lengths $\xi$, were estimated using the least-squares approach. Results are listed in Table 5. Italic values indicate situations, where the least-squares estimates resulted in the improper values as the negative variances and the unrealistic correlation lengths. The analytical covariance function CF6 for $T_{Z Z}$ component approximating empirical covariances is shown in Fig. 4. In compliance with the coefficient of determination, the analytical function CF6 accounts better performance. The highest variances are again typical for the sub-region R2 and the correlation lengths both for all evaluated components and the sub-regions vary between 103 and $195 \mathrm{~km}$ (only for CF6 model). This implies that covariance functions are highly responsive to the area of calculation and are, of course, different for particular components of disturbing gravitational tensor.

\section{Interpolation of SGG data using least-squares collocation}

The best way to demonstrate usefulness of the covariance function is to interpolate SGG data in order to produce regular local grid of disturbing gravitational gradients. For this purpose, the least-squares collocation (LSC) method has been used, where the covariance function plays an essential role. More details are explained e.g. in Moritz and Sünkel (1978), Moritz (1980).

Our experiment is restricted to the sub-region R1 and the largest component $T_{Z Z}$ measured from 1 September 2013 to 19 October 2013. The regular grid with the resolution of $0.1^{\circ} \times 0.1^{\circ}$ is located on the spherical surface with the radius of $R_{1}=6595 \mathrm{~km}$. LSC enables to adjust both the trend of the disturbing gravitational gradients and the signal, which was modeled by the 2-dimensional covariance function CF6 with the estimated variance $C_{0}=0.086 \mathrm{E}^{2}$ and the correlation length $\xi=160.1 \mathrm{~km}$ (see Table 5). We have considered the constant trend related to a systematic part of disturbing gravitational gradients in the tested sub-region and uncorrelated errors of observations corresponding to the

Table 5 The estimated parameters of local covariance models CF5 and CF6 (the 2-dimensional case)

\begin{tabular}{|c|c|c|c|c|c|c|c|c|c|}
\hline \multirow[t]{2}{*}{ Sub-reg. } & \multirow[t]{2}{*}{ Param. } & \multicolumn{2}{|l|}{$T_{X X}$} & \multicolumn{2}{|l|}{$T_{Y Y}$} & \multicolumn{2}{|l|}{$T_{Z Z}$} & \multicolumn{2}{|l|}{$T_{X Z}$} \\
\hline & & CF5 & CF6 & CF5 & CF6 & CF5 & CF6 & CF5 & CF6 \\
\hline \multirow[t]{4}{*}{ R1 } & $C_{0}\left(\mathrm{E}^{2}\right)$ & 0.028 & 0.023 & 0.056 & 0.042 & 0.107 & 0.086 & 0.045 & 0.039 \\
\hline & $\xi(\mathrm{km})$ & 106.6 & 167.2 & 83.8 & 146.9 & 98.2 & 160.1 & 132.2 & 194.5 \\
\hline & $R s q(\%)$ & 87.4 & 95.8 & 85.7 & 94.6 & 84.8 & 94.4 & 79.8 & 89.1 \\
\hline & $C_{0}\left(\mathrm{E}^{2}\right)$ & 0.488 & 0.403 & 0.126 & 0.105 & -0.330 & 0.724 & 0.531 & 0.469 \\
\hline \multirow[t]{3}{*}{$\mathrm{R} 2$} & $\xi(\mathrm{km})$ & 105.1 & 164.1 & 102.7 & 152.3 & -292.0 & 159.9 & 135.4 & 185.2 \\
\hline & $R s q(\%)$ & 80.0 & 90.7 & 87.9 & 95.0 & 54.5 & 91.0 & 83.7 & 93.5 \\
\hline & $C_{0}\left(\mathrm{E}^{2}\right)$ & 0.119 & 0.025 & -0.008 & 0.013 & 5.324 & 0.049 & -0.076 & 0.038 \\
\hline \multirow[t]{3}{*}{ R3 } & $\xi(\mathrm{km})$ & 13.6 & 105.4 & -99.8 & 120.1 & 0.6 & 103.1 & -30.1 & 110.9 \\
\hline & $R s q(\%)$ & 85.5 & 92.8 & 82.6 & 72.0 & 85.0 & 91.9 & 84.7 & 89.1 \\
\hline & $C_{0}\left(\mathrm{E}^{2}\right)$ & 0.024 & 0.020 & 0.016 & 0.010 & 0.052 & 0.045 & 0.047 & 0.034 \\
\hline \multirow[t]{2}{*}{ R4 } & $\xi(\mathrm{km})$ & 125.2 & 181.8 & 61.1 & 136.6 & 130.0 & 181.5 & 78.0 & 144.1 \\
\hline & $R s q(\%)$ & 84.8 & 93.5 & 85.0 & 93.9 & 88.3 & 95.9 & 79.5 & 88.0 \\
\hline
\end{tabular}


accuracy of $T_{Z Z}$ component given in EGG_TRF_2 products for SGG data. Figure 5a shows directly interpolated disturbing gravitational gradients $T_{Z Z}$ in the sub-region R1 applying Delaunay triangulation method implemented in GMT 5 software (Wessel et al. 2013), which was used for producing figures. Using LSC, we have obtained smoothed disturbing gravitational gradients in Fig. $5 \mathrm{~b}$ interpolated in the regular grid with the given resolution and the suppressed random noise.

Proving the reliability of the local grid obtained by LSC, we have made the comparison with the corresponding set of grids produced by the Earth's global gravity field models. We have used three recent models: TIM R5 up to d/o 200 (Brockmann et al. 2014), EIGEN6C4 up to d/o 200 and 300 (Förste et al. 2014), and EGM2008 up to d/o 200 and 300 (Pavlis et al. 2012). TIM R5 is purely determined from the GOCE observations and is based on the time-wise approach. EIGEN-6C4 is a combined gravity field model using different satellite (LAGEOS, GRACE, and GOCE) and terrestrial data sets. EGM2008 was formed by combination of satellite, terrestrial, altimetry and airborne gravity data. Grids from these models were generated in 'GrafLab' software (Bucha and Janák 2013) in the altitude corresponding to the radial distance $R_{1}=6595 \mathrm{~km}$ and with the same resolution as the produced LSC grid.

The basic summary statistics from the Earth's global gravity field models are listed in Table 6 (upper part). For completeness, the residuals between model grids and the interpolated LSC grid are presented. Residuals vary between $-0.043 \mathrm{E}$ and $0.030 \mathrm{E}$ with the mean close to zero, see the lower part of Table 6 . The best fit is obtained by applying EIGEN-6C4 model (d/o 300) with the standard deviation of 0.0047 E. It might indicate that there is still some useful signal in our solution corresponding to frequencies above d/o 200, and certain part of discrepancies is due to the omission error and noise. We recall that EIGEN-6C4 also includes GOCE data. The lowest agreement is evident for EGM2008 (d/o 200). However, discrepancies between compared models are negligible in most cases.

Let us look at the comparison with EIGEN-6C4 model (d/o 300) having the best fit more closely. Disturbing gravitational gradients obtained from this model are shown in Fig. $6 \mathrm{a}$ and corresponding residuals in Fig. $6 \mathrm{~b}$. It is clearly visible that anomalous residuals mostly occur close to the limits of the sub-region R1. This effect is caused by missing information about the gravity field outside the sub-region, which has an influence on the covariance function construction and the interpolation procedure. Almost $99 \%$ of residuals

(a)

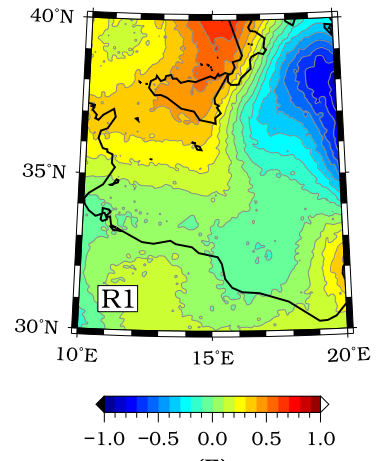

(E) (b)

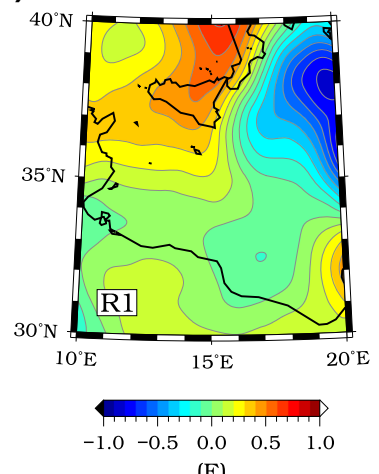

(E)

Fig. 5 Disturbing gravitational gradients $T_{Z Z}$ : a directly interpolated from 'measured' gradients using Delaunay triangulation method, $\mathbf{b}$ interpolated in the regular grid of $0.1^{\circ} \times 0.1^{\circ}$ applying $\operatorname{LSC}$ 
Table 6 The basic statistics of the Earth's global gravity field models (upper part) and their comparison with LSC grid (lower part) in the sub-region R1 for $T_{Z Z}$ component

\begin{tabular}{lcclc}
\hline Grid/model & MEAN (E) & MIN (E) & MAX (E) & STD (E) \\
\hline LSC & 0.0586 & -0.8506 & 0.7087 & 0.2788 \\
TIM R5 (d/o 200) & 0.0579 & -0.8447 & 0.7058 & 0.2792 \\
EIGEN-6C4 (d/o 200) & 0.0580 & -0.8447 & 0.7046 & 0.2791 \\
EIGEN-6C4 (d/o 300) & 0.0580 & -0.8483 & 0.7080 & 0.2792 \\
EGM2008 (d/o 200) & 0.0580 & -0.8436 & 0.7071 & 0.2794 \\
EGM2008 (d/o 300) & 0.0579 & -0.8474 & 0.7103 & 0.2794 \\
\hline TIM R5 (d/o 200) - LSC & -0.0007 & -0.0395 & 0.0266 & 0.0049 \\
EIGEN-6C4 (d/o 200) - LSC & -0.0006 & -0.0395 & 0.0272 & 0.0050 \\
EIGEN-6C4 (d/o 300) - LSC & -0.0006 & -0.0397 & 0.0292 & 0.0047 \\
EGM2008 (d/o 200) - LSC & -0.0007 & -0.0422 & 0.0279 & 0.0060 \\
EGM2008 (d/o 300) - LSC & -0.0007 & -0.0425 & 0.0296 & 0.0058 \\
\hline
\end{tabular}

does not exceed the value of $0.01 \mathrm{E}$, what also confirms the centered histogram of residuals in Fig. 6c. If we calculate the summary statistics of residuals from the restricted sub-region $\mathrm{R} 1$, which is reduced to $\phi \in\left\langle 32^{\circ} \mathrm{N}, 38^{\circ} \mathrm{N}\right\rangle$ and $\lambda \in\left\langle 12^{\circ} \mathrm{E}, 18^{\circ} \mathrm{E}\right\rangle$, we will get more optimistic values: $\quad \mathrm{MEAN}=-0.0006 \mathrm{E}, \quad \mathrm{MIN}=-0.0125 \mathrm{E}, \quad \mathrm{MAX}=0.0102 \mathrm{E}, \quad$ and $\mathrm{STD}=0.0037 \mathrm{E}$. In comparison with Table 6, improvement of the standard deviation is more than $20 \%$. This implies that a local grid should be created using a covariance function derived from an extended area, where more information about the surrounding Earth's gravity field is included.

(a)

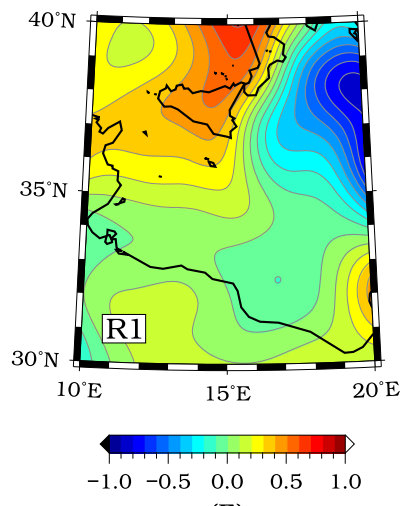

(E) (b)

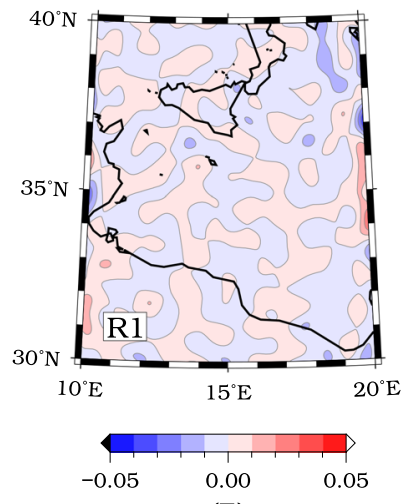

(E) (c)

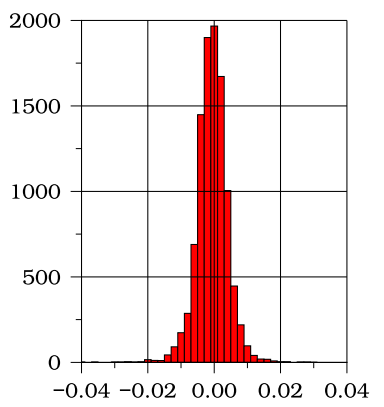

(E)

Fig. 6 Disturbing gravitational gradients $T_{Z Z}$ : a from the Earth's global gravity field model EIGEN-6C4 (up to d/o 300), b residuals between EIGEN-6C4 model (up to d/o 300) and LSC grid, and c the histogram of residuals 


\section{Conclusions}

An analysis of local covariance functions of disturbing gravitational gradients derived from EGG_TRF_2 products was carried out in this study. Only covariance functions in the planar approximation were considered. Firstly, we have investigated four different types of the 1-dimensional covariance functions. In this situation, we assumed all data points are located on the approximation sphere, which is not exactly fulfilled for SGG data. The first numerical experiment reveals that the most appropriate from analytical covariance functions is the Gaussian function CF1 showing the best performance in all sub-regions. The parameters of the covariance function differ from region to region significantly. In our case of four adjacent sub-regions and $T_{\mathrm{ZZ}}$ component, the variance vary from $0.024 \mathrm{E}^{2}$ to $0.412 \mathrm{E}^{2}$ and the correlation length from 137 to $214 \mathrm{~km}$. The lower correlation length indicates that the function is in average relatively more dissected. In addition, a time stability of essential parameters was studied. A sensitivity of estimated parameters to the changes of GOCE satellite orbit during various measurement cycles was showed. It appears that a correct modeling of stochastic dependencies between the observed gravity gradients requires evaluation of essential parameters directly from the region of interest. In general, the local covariance models with longer correlation length are more suitable for satellite gravity gradiometry data due to the smoothness of Earth's gravity field.

Problem with the altitude variation of GOCE orbit can be treated by using the 2-dimensional covariance function. Moreover, this enables us to perform the downward continuation and the interpolation of measurements in one step. Presented procedure takes into account different altitudes of data points above the approximation sphere. We have studied two types of the 2-dimensional analytical functions. For all tested sub-regions, the covariance function CF6 shows better performance and numerical stability. Analysis showed again that estimated parameters are highly responsive to the input data in particular sub-region. The highest variances are typical for the sub-region R2 with the maximum signal amplitudes.

The 2-dimensional covariance model CF6 was applied to the interpolation of disturbing gravitational gradients $T_{Z Z}$ into the regular grid using the least-squares collocation method. The local grid at the altitude corresponding to the radial distance of $R_{1}=6595 \mathrm{~km}$ was compared to values obtained from the recent Earth's global gravity field models. We have used three recent models: TIM R5 up to d/o 200, EIGEN-6C4 up to d/o 200 and 300, and EGM2008 up to d/o 200 and 300. The best fit was achieved by applying EIGEN-6C4 model (d/o 300) with the mean of $-0.0006 \mathrm{E}$ and the standard deviation of $0.0047 \mathrm{E}$. A cause of this may be the fact that this model also includes GOCE data. Disagreement of the interpolated grid with the model values mostly occurs near the limits of the sub-region, from which the covariance function was constructed. Omitting the $2^{\circ}$ stripes around the limits of the sub-region brings improvement of the standard deviation of residuals more than $20 \%$. The obtained results show practicability of the local covariance functions in planar approximation, ignoring the Earth's sphericity, for solving the detail problems related to modeling of Earth's gravity field using satellite gravity gradiometry data. However, we must underline the fact that local grids computed following this approach are mostly intended for geophysical research and local applications.

Acknowledgements We gratefully acknowledge the reviewers comments and suggestions, which improved the paper essentially. This study is based on research carried out within the Slovak National Project VEGA 1/0954/15: Analysis of Global Data Sources and Possibilities of Their Application in the Refinement and Testing of Earth Gravity Field Models. Thanks also to the HPC center at the Slovak University of 
Technology in Bratislava, which is a part of the Slovak Infrastructure of High Performance Computing (SIVVP project, ITMS code 26230120002, funded by the European region development funds, ERDF), for the computational time and resources made available.

\section{References}

Bouman J, Ebbing J, Fuchs M, Sebera J, Lieb V, Szwillus W, Haagmans R, Novak P (2016) Satellite gravity gradient grids for geophysics. Nat Sci Rep. doi:10.1038/srep21050

Brockmann JM, Zehentner N, Höck E, Pail R, Loth I, Mayer-Gürr T, Schuh WD (2014) EGM_TIM_RL05: an independent geoid with centimeter accuracy purely based on the GOCE mission. Geophys Res Lett 41:8089-8099. doi:10.1002/2014GL061904

Bucha B, Janák J (2013) A MATLAB-based graphical user interface program for computing functionals of the geopotential up to ultra-high degrees and orders. Comput Geosci 56:186-196

ESA (1999) Gravity field and steady-state ocean circulation mission, report for mission selection of the four candidate Earth explorer missions. Technical report ESA SP-1233(1), European Space Agency, ESA Publication Division

Förste C, Bruinsma S, Abrikosov O, Lemoine JM, Marty JC, Flechtner F, Balmino G, Barthelmes F, Biancale R (2014) EIGEN-6C4 the latest combined global gravity field model including GOCE data up to degree and order 2190 of GFZ Potsdam and GRGS Toulouse. doi:10.5880/icgem.2015.1. Accessed 12 Feb 2014

Gatti A, Migliaccio F, Reguzzoni M, Sansò F (2013) Space-wise global grids of GOCE gravity gradients at satellite altitude. In: Geophysical research abstracts. Abstracts of the earth, planetary and space sciences, vol 15. Copernicus Publications, Göttingen

Gruber T, Rummel R, Abrikosov O, Van Hess R (2010) GOCE level 2 product data handbook. Technical report GO-MA-HPF-GS-0110, The European GOCE Gravity Consortium EGG-C

Heiskanen WA, Moritz H (1967) Physical Geodesy. W. H, Freeman and Company, San Francisco

Jekeli C (2010) Correlation modeling of the gravity field in classical geodesy. Springer, Berlin, pp 833-863. doi:10.1007/978-3-642-01546-5_28

Moritz H (1980) Advanced physical geodesy. Wichmann, Karlsruhe

Moritz H, Sünkel H (1978) Approximation methods in geodesy. Wichmann, Karlsruhe

Pail R, Bruinsma S, Migliaccio F, Förste C, Goiginger H, Schuh WD, Höck E, Reguzzoni M, Brockmann JM, Abrikosov O, Veicherts M, Fecher T, Mayrhofer R, Krasbutter I, Sansò F, Tscherning CC (2011) First GOCE gravity field models derived by three different approaches. J Geodesy 85:819-843. doi:10. 1007/s00190-011-0467-x

Pavlis NK, Holmes SA, Kenyon SC, Factor JK (2012) The development and evaluation of the earth gravitational model 2008 (EGM2008). J Geophys Res. doi:10.1029/2011JB008916

Rapp R (1978) Results of the application of least-squares collocation to selected geodetic problems. In: Moritz H, Sünkel H (eds) Approximation methods in geodesy. Wichmann, Karlsruhe, pp 117-156

Rousseeuw PJ, Leroy AM (1987) Robust regression and outlier detection. Wiley, New York

Šprlák M (2012) A graphical user interface application for evaluation of the gravitational tensor components generated by a level ellipsoid of revolution. Comput Geosci 46:77-83

Tscherning CC (1976) Covariance expressions for second and lower order derivatives of the anomalous potential. Technical report 225, Department of Geodetic Science, The Ohio State University

Tscherning CC (2010) The use of least-squares collocation for the processing of GOCE data. Vermess Geoinf 1:21-26

Wessel P, Smith WHF, Scharroo R, Luis J, Wobbe F (2013) Generic mapping tools: improved version released. Eos Trans Am Geophys Union 94(45):409-410. doi:10.1002/2013EO450001 